\title{
A novel microduplication in INPP5A segregates with Schizophrenia Spectrum Disorder in the family of a patient with both Childhood Onset Schizophrenia and Autism
}

\section{Spectrum Disorder}

Arnaud Fernandezi,2,3, Małgorzata Drozd ${ }^{3}$, Susanne Thümmler ${ }^{1,2}$, Barbara Bardoni ${ }^{3,4}$, Florence Askenazy ${ }^{1,2 *}$, Maria Capovilla ${ }^{3 * \#}$

1) Departement de Psychiatrie de l'Enfant et de l'Adolescent Hôspital de NICE CHU-Lenval, 06200 Nice, France

2) CoBTek, FRIS, Université Côte d'Azur, Nice, France

3) Université Côte d'Azur, CNRS UMR7275, Institut de Pharmacologie Moléculaire et Cellulaire, 660 Route des Lucioles, Valbonne, France

4) Université Côte d'Azur, INSERM, CNRS UMR7275, Institut de Pharmacologie Moléculaire et Cellulaire, 660 Route des Lucioles, Valbonne, France

* To whom correspondence should be addressed: maria.capovilla@ipmc.cnrs.fr \# Equal contribution 


\begin{abstract}
Childhood-Onset Schizophrenia (COS) is a very rare and severe psychiatric disorder defined by adult schizophrenia symptoms occurring before the age of 13 . We report a microduplication in the 10q26.3 region including part of the Inositol Polyphosphate-5Phosphatase $A$ (INPP5A) gene that segregates with Schizophrenia Spectrum Disorders (SSDs) in the family of a female patient affected by both COS and Autism Spectrum Disorder (ASD). Phenotyping and genotyping (including CGH-array) were performed for mother, healthy sister and affected child according to the GenAuDiss protocol (NCT02565524). The duplication size is $324 \mathrm{~kb}$ and is present in a patient with COS and in her mother with SSD, but not in the patient's healthy sister. INPP5A encodes a membrane-associated $43 \mathrm{kDa}$ type I inositol 1,4,5-trisphosphate (InsP3) 5-phosphatase. This protein is found both in mouse and human brains and we found that its Drosophila homologue 5PtaseI is specifically expressed in the central nervous system. Hydrolyzed products from InsP3 5-phosphatases mobilize intracellular calcium, which is relevant for dendritic spine morphogenesis in neurons, altered in both schizophrenia and ASD. These may constitute arguments in favor of this gene alteration in the pathophysiology of COS.
\end{abstract}

Keywords: Childhood-Onset Schizophrenia, neurodevelopmental disorder, INPP5A, CNV, inositol pathway. 


\section{Introduction}

Schizophrenia spectrum disorders (SSDs) include schizophrenia, other psychotic disorders and schizotypal (personality) disorder [1]. Childhood-Onset Schizophrenia (COS) is a very rare and severe form of schizophrenia defined by Adult-Onset Schizophrenia (AOS) symptoms occurring before the age of 13 according to the Diagnostic and Statistical Manual of mental disorders $5^{\text {th }}$ edition (DSM-5): disorganized speech and behavior, hallucinations and delusions [1-2]. This disorder is highly heterogenic at both clinical and genetic levels. As in other neurodevelopmental disorders, the pathophysiology of COS involves genetic factors as well as environmental factors damaging the brain (e.g., hypoxemia, early childhood trauma or maternal-fetal infections) [3]. The respective responsibility of each of its causes remains unknown [4]. The genetic architecture of COS is complex and involves DNA changes: Copy Number Variations (CNVs) and Single Nucleotide Polymorphisms (SNPs), both inherited and de novo [5 - 8]. At a metabolic level, many different pathways have been implicated in the pathogenesis of schizophrenia and alterations in the phosphoinositide signaling pathway leading to changes in $\mathrm{Ca}^{2+}$ signaling play a major role [9]. According to the $N$-Methyl-DAspartate Receptor (NMDAR) hypo-function hypothesis of schizophrenia pathogenesis, the decline in $\mathrm{Ca}^{2+}$ signaling resulting from a decrease in NMDAR activity is responsible for the instability of the GABAergic inhibitory neurons [10]. These neurons express receptors that activate the phosphoinositide signaling pathway acting to increase the $\mathrm{Ca}^{2+}$ signal and maintaining an excitatory/inhibitory balance [9].

We present the description of a microduplication in the 10q region including part of the Inositol Polyphosphate-5-Phosphatase A (INPP5A; HGNC: 6076/Entrez Gene ID: 3632/ OMIM: 600106/Ensembl: ENSG00000068383) gene that segregates with SSDs in the family of a patient affected by both COS and Autism Spectrum Disorder (ASD). We also show the 
expression of the INPP5A Drosophila homologue in the central nervous system, supporting its role in the pathogenesis of COS.

\section{Materials and Methods}

\section{GenAuDiss protocol}

GenAuDiss is a translational multicentric study (NCT02565524). Patients with COS or EarlyOnset-Schizophrenia (EOS) (with symptoms occurring before the age of 18) were initially recruited through the 2011-2013 Interregional Hospital Clinical Research Program (NCT01512641) whose main goal was to estimate the prevalence of COS and EOS in a population of children and adolescents in child psychiatry care or medical-educational structures $[11,12]$. In addition, an enrolment in the present study is offered to inpatients and outpatients of Child and Adolescent Psychiatry (CAP) centers, as well as their first-degree relatives. Participants are included either directly at the study sites or after referral by child or adolescent psychiatrists of the Provence-Alpes-Côte d'Azur (PACA) region. At inclusion (V1), clinical assessments (both psychiatric and neurocognitive) are performed in patients as well as first-degree relatives (parents and siblings). Blood tests are performed in a second visit (V2) within six months.

The study is proposed to children and adolescents presenting COS or EOS with comorbid ASD and to their first-degree relatives. Major inclusion criteria for patients:

- 7-22 years of age;

- Diagnosis of COS (onset before age 13) or EOS (onset before age 18) by the semistructured interview Kiddie Schedule for Affective Disorders and SchizophreniaPresent and Lifetime Version (K-SADS-PL) psychosis section; 
- Diagnosis of ASD by Autism Diagnostic Interview-Revised (ADI-R);

- IQ $\geq 40$ at Wechsler Intelligence Scale for Children (WISC-V).

Major inclusion criteria for parents and siblings:

- First-degree biological relationship (no adoption) with the index patient.

The main objective of the GenAuDiss protocol is to identify disease-causing genetic mutations in a cohort of patients affected by both COS or EOS and ASD.

\section{Phenotyping}

The propositus is an inpatient at the time of the inclusion process. Quantitative and qualitative measures were assessed during hospitalization:

- Medical history (including pregnancy, birth and later development) and biographic parameters, types and dates of significant life events including trauma and environmental exposures (e.g., drugs and substances);

- Clinical parameters from physical examination (e. g., body weight, body mass index and arterial pressure);

- Semistructured interviews to assess main diagnosis at inclusion [K-SADS-PL, ADI-R and Mini International Neuropsychiatric Interview (MINI)][13-16]);

- Clinical heteroassessments with specific rating scales [WISC-V, Positive and Negative Syndrome Scale (PANSS), Scale for the Assessment of Negative Symptoms (SANS), Trail Making Test (TMT) A, TMT B, and verbal fluency] [17-20];

- Self-report questionnaires [Cloninger's Temperament and Character Inventory (TCI) 226 and Baron Cohen's Autism Quotient (AQ)] [21-23].

Genotyping 
The genetic analysis strategy includes DNA testing for Fragile-X Syndrome by PCR FRAXA [24], high-resolution karyotype or DNA CNV research by CGH array from both parents, siblings and patient. A quantitative PCR (LightCycler ${ }^{\circledR} 480$ SYBR Green I Master) is made for gene detection and quantification if a putative gene is found to be deleted, duplicated or truncated by a CNV.

In situ hybridization in Drosophila

Drosophila embryos were dechorionated in $50 \%$ bleach, fixed in $4 \%$ formaldehyde, devitellinized in $100 \%$ methanol, and stored in $100 \%$ ethanol. The 5 PtaseI anti-sense probe was made from the Drosophila Genomics Resource Center (DGRC) SD05875 cDNA linearized with EcoRI and transcribed with $\mathrm{T} 7$ polymerase using the Riboprobe Combination System kit (Promega, Madison, WI) and the DIG RNA Labeling Mix (Roche, Indianapolis, IN). Embryos were stained using the TSA Plus Biotin System (Perkin Elmer, Waltham, MA) according to Tevy et al., 2014 [25].

\section{Results}

The GenAuDiss protocol [11], a French multicentric study designed to identify diseasecausing genetic mutations in a cohort of patients affected by COS or EOS and ASD, allowed us to identify a novel interesting CNV in a COS proband: a $\sim 324 \mathrm{~kb}$ duplication including a portion of the INPP5A gene. Mitchell et al. (1996) used fluorescence in situ hybridization to map the gene on chromosome 10 (10q26.3) [26]. The duplication was inherited from the mother diagnosed with psychotic disorder that is included in SSD. The healthy sister of the 
patient does not carry this duplication (Figure 1). Unfortunately, the father died at patient's early childhood, is not available for genetic testing and we do not have any psychiatric information about him.

\section{Medical history and developmental features}

The proband, a 17-year-old girl, was diagnosed with COS around age 12. She was born at 41 weeks of gestation, by caesarian section. Her birthweight was $3500 \mathrm{~g}$, she measured $51 \mathrm{~cm}$ of length and $36 \mathrm{~cm}$ of head circumference. Apgar score was normal (10-10). She presents a psychotraumatic family history with the violent father's death murdered at her early childhood (before one year old). Delayed motor milestones were noticed (walking after 24 months) with also communication disorders (first words at three and a half years and stuttering), intellectual disability (IQ = 74) and learning impairments. She was admitted in a medical-social care center since the age of seven years, because of behavioral symptoms including tantrums and poor interactions with other children. At age 12, she presented a delusion of persecution, visual hallucinations and depressive mood and was diagnosed with COS by two independent child psychiatrists. She was treated by a monotherapy of antipsychotic (Risperidone) during five years with a good response on delusion, but mood lability and depressive symptoms remained. At the age of 14 , she made a first suicide attempt by drug ingestion. During hospitalization in an adolescent psychiatry unit, a switch to Olanzapine allowed a durable mood stabilization with also a good response for positive symptoms (delusions and hallucinations).

\section{Clinical work-up}


At the age of 17 , she was hospitalized again for a relapse of suicidal behavior with associated mood-congruent psychotic features. Neurological and general examinations were normal, including EEG, cerebral MRI, standard blood analysis, and metabolic screening.

\section{Somatic features}

The patient has been and is presently suffering from moderate asthma (no long-term control medications needed), primary enuresis and primary amenorrhea (first periods at the age of 17) without associated drug-induced hyperprolactinemia.

\section{Clinical Genetics}

Fragile-X Syndrome testing by PCR FRAXA was negative. High-resolution karyotype was not carried out because we first performed a molecular karyotype (Array CGH).

Array CGH analysis shows an interstitial $324 \mathrm{~kb}$ duplication in the chromosomal region 10q26.3, inherited from the mother (Figure 2A, B) that was confirmed by Quantitative PCR (qPCR). Three genes are contained in this microduplication: AF086154, LINC02870 and INPP5A. Only one gene (INPP5A) is expressed in the brain, The other two genes have unknown function and no data about the encoded proteins are available (Figure 2C). Only one portion of INPP5A is duplicated in this interval (transcript ENST00000368593.7: from exon 1/13 to $12 / 13$ with truncation in intron 12/12; transcript ENST00000368594.8: from exon 1/16 to $12 / 16$ with truncation in intron $12 / 15$; transcript ENST00000342652.6 : from exon $1 / 13$ to $9 / 13$ with truncation in intron $9 / 12$ ).

\section{Family study}

The patient's mother was evaluated with MINI-S and was diagnosed with psychotic disorder, mood/anxiety disorders (suicide ideation without suicide attempt) and Post-Traumatic Stress Disorder (PTSD). The AQ was high (30/50) and revealed autistic traits, near the cut-off 
(32/50) for Asperger's syndrome diagnosis. The TCI personality test revealed also a probable personality disorder (cooperation <-2 SD). Finally, IQ was normal around 108. In her medical history, she felt sad very regularly but had never seen a psychiatrist and had never benefited from a psychotropic drug. The patient's sister was diagnosed with tobacco use disorder and past social phobia. Her AQ score was negative for autistic traits $(16 / 50)$ and no personality disorder was diagnosed. Her medical history was insignificant.

\section{Central nervous system expression in Drosophila}

The human INPP5A gene has one Drosophila homologue called 5PtaseI [25]. The two proteins present $46 \%$ identity and $63 \%$ similarity. No 5PtaseI mutant is currently available and its expression pattern has not been reported. To determine whether the function of INPP5A is conserved, we carried out in situ hybridization on Drosophila embryos. At stages 15 and 16, 5PtaseI is strongly expressed in the brain and in the ventral nerve cord (Appendix 1). This result reinforces the role of INPP5A in nervous system development.

\section{Discussion}

Our case report is the description of a patient with COS associated with neurodevelopmental disorders (mental retardation, leaning disabilities and ASD) and carrying a 10q26.3 micromicroduplication in the INPP5A gene. According to the large Decipher database (https:// decipher.sanger.ac.uk/), most gain and loss of function CNVs encompassing INPP5 are predicted to be pathogenic in the general population. In the patient's family, the genetic testing of first degree relatives (Figure 1) revealed a familial segregation of the mutation 
where both mother and patient with SSD carry this 10q26.3 CNV microduplication while the 22 year-old sister in good mental health (with typical development) does not carry it.

While the proband has an earlier, very severe and neurodevelopmental form of schizophrenia, her mother's psychotic disorder evolved since adolescence (15 years). We can speculate that a second "hit" is present (e.g., an additional genomic mutation or an epigenetic effect of early childhood trauma). Common variants (SNPs carried by more than $1 \%$ of the general population) may also play a role in the pathogenesis of COS when added to rare mutations [5].

INPP5A encodes a membrane-associated $43 \mathrm{kDa}$ type I inositol 1,4,5-trisphosphate (InsP3) 5-phosphatase that hydrolyzes $\operatorname{Ins}(1,4,5) \mathrm{P} 3$ and $\operatorname{Ins}(1,3,4,5) \mathrm{P} 4$ to generate $\operatorname{Ins}(1,4) \mathrm{P} 2$ and $\operatorname{Ins}(1,3,4) \mathrm{P} 3$, respectively [27, 28]. InsP3 5-phosphatases hydrolyze $\operatorname{Ins}(1,4,5) \mathrm{P} 3$, which mobilizes intracellular calcium and acts as a second messenger mediating cell responses to various stimulation [9]. Primary and modulatory actions of the $\mathrm{Ins} \mathrm{P}_{3} / \mathrm{Ca}^{2+}$ pathway have been implicated in psychiatric disorders (ASD, schizophrenia and bipolar disorder) and in major human diseases affecting the brain (Alzheimer's, Huntington's diseases, amyotrophic lateral sclerosis, epilepsy, and spinocerebellar ataxia) or other tissues (e.g., cardiac diseases) [9]. This protein is found in both mouse [29; http://mouse.brain-map.org/gene/show/84380] and human brains [30; https://www.genecards.org/cgi-bin/carddisp.pl?gene=INPP5A\#expression]. Phosphoinositides are phospholipids participating in membrane remodeling and all the processes linked to it, like cytoskeletal remodeling, cell signaling cascades, ion channel activity, and membrane traffic [31]. At a molecular level, INPP5A appears to regulate intracellular $\mathrm{Ca}^{2+}$ through modulation of $\operatorname{Ins}(1,4,5) \mathrm{P} 3$ levels, which is relevant for dendritic spine morphogenesis in neurons [32-34]. Importantly, dendritic spine morphology and 
number are altered in both schizophrenia [35] and ASD [36]. These may constitute arguments in favor of the role of this gene alteration in the pathophysiology of COS.

Furthermore, it would be interesting to explore the levels, in both mother and daughter, of the truncated InsP3 5-phosphatase that we hypothesize is generated by the microduplication. Indeed, the presence of an abnormal InsP3 5-phosphatase would strongly suggest a dominant-negative effect. However, due the inaccessibility of the tissues where InsP3 5-phosphatase is expressed, this important information is lacking.

Lithium, a classic mood stabilizer in clinical practice, reduces the formation of inositol (by inositol monophosphatase inhibition) and seems to be a therapeutic target of this pathway [9]. Reduced brain inositol in rats treated by lithium was first shown more than 50 years ago [37]. Berridge et al., (1989) introduced the inositol depletion hypothesis to explain the clinical effects of lithium, which postulates a reduction in the supply of inositol lipids required for signaling in cells within the CNS (therapeutic action) or in the developing embryo (teratogenic action) [38]. More recently, a growing literature about the neuroprotective action of lithium in various CNS disorders including neurodegenerative disorders is emerging [39]. However, despite a suggested link between the alteration in the inositol pathway (INPP5A microduplication) and the diagnosis of our patient, the therapeutic effects of lithium on COS and other forms of schizophrenia remain unknown and should be specifically studied.

In mice, the loss of function of Inpp5A (presenting 90,21\% similarity with human INPP5A) causes ataxia and cerebellar Purkinje cell degeneration [29]. In humans, INPP5A has been shown to play a role in the development and progression of cutaneous squamous cell carcinoma and to have a prognostic value in these diseases [40, 41]. Strong proof of the involvement of the brain-expressed INPP5A gene in human psychiatric disorders is lacking. 
To our knowledge, only two SNVs (rs751524528: p.A80V and rs775793924: p.R168W) were described in human psychiatric disorders. rs751524528 (p.A80V) is a benign nonsynonymous mutation (according to Polyphen2) in a 31-year-old female affected by adultonset schizoaffective disorder (bipolar type) without developmental delays, mental retardation and learning disabilities [42]. rs775793924 (p.R168W) is a rare SNV defined by authors as a «non-synonymous damaging strict and disruptive» variant and was found in a multiplex family with subjects affected by bipolar disorder or SSD with no phenotypic description [43]. Finally, an epigenome-wide significant $\left(P<1.7 \times 10^{-8}\right)$ association for phonemic verbal fluency $\left(\operatorname{cg} 12507869, P=2.5 \times 10^{-9}\right)$ was found in an epigenome-wide association study. This CpG site is located in INPP5A, but the top SNP (rs113565688) for this site explains only $1.2 \%$ of the variance in methylation and rs113565688 was not correlated with alterations in cognitive functions in a recent GWAS. Thus, the environmental influence in INPP5A methylation in the cognitive alteration seems predominant over the genetic contribution [44].

In Drosophila, all the genes of the phosphoinositol pathway are present except for the $\mathrm{I}(1,3,4) \mathrm{P}_{3} 5 / 6$ kinase [45]. The INPP5A homolog 5PtaseI is among the Drosophila genes with high probability of having a synaptic function [46] and it is a robust suppressor of Ataxin-3 toxicity in the eye [47]. 5PtaseI is a modifier of toxicity brought up by expression of expanded PolyQ Ataxin-3 present in Spino Cerebellar Ataxia 3 (SCA3) patients [47]. This was found through a deep RNAi screen in the eye and shown to involve protein turnover/ quality control, nuclear import/export and mRNA transport/editing/translation. In Drosophila, the INPP5A homologue is a crucial enzyme in the phosphoinositide pathway. We found that 5PtaseI is expressed exclusively in the central nervous system of embryos. This original finding suggests that INPP5A is a gene involved in central nervous system development. 
Furthermore, we can propose that the fly may be an excellent model to study the role of INPP5A/5PtaseI in normal and pathological development of the nervous system.

In clinical practice, as suggested by Szego et al. for ASD [48], it would seem useful to propose to COS patients systematic whole exome/genome sequencing instead of or in addition to microarrays to improve genetic testing, allow SNV detection and consequently end the "diagnostic Odyssey" [49]. In research, the major challenge of the upcoming years will be the analysis of big data from next generation sequencing (prioritization and interpretation of DNA variations) [50] and the experimental validation of putative mutations in animal models. Sharing patients' data with other teams around the world will be helpful to unravel the molecular pathophysiology of COS and ASD and their underlying causes, paving the way for an early therapeutic intervention.

\section{Acknowledgments}

This study is supported by CNRS, INSERM and Monaco Against Autism (MONAA). MD was supported by the LabEx "Signalife" international PhD program ANR-11-LABX-0028-01. We are grateful to Angela Algeri and Gaëlle Laure for comments on the manuscript. We thank Franck Aguila for graphical help.

\section{Data Availability Statement}

The data that support the findings of this study are available on request from the corresponding author. The data are not publicly available due to privacy or ethical restrictions.

\section{References}


1) Association AP. Diagnostic and statistical manual of mental disorders (DSM-5®): American Psychiatric Pub; 2013.

2) Nicolson R, Rapoport JL. Childhood-onset schizophrenia: rare but worth studying. Biol Psychiatry. 1999;46(10):1418-28.Rapoport J, Giedd J, Gogtay N. Neurodevelopmental model of schizophrenia: update 2012. Molecular psychiatry. 2012;17(12):1228-38.

3) Davis J, Eyre H, Jacka FN, Dodd S, Dean O, McEwen S, et al. A review of vulnerability and risks for schizophrenia: beyond the two hit hypothesis. Neuroscience \& Biobehavioral Reviews. 2016;65:185-94.

4) Addington AM, Rapoport JL. The genetics of childhood-onset schizophrenia: when madness strikes the prepubescent. Current psychiatry reports. 2009;11(2):156-61.

5) Ahn K, Gotay N, Andersen T, Anvari A, Gochman P, Lee Y, et al. High rate of diseaserelated copy number variations in childhood onset schizophrenia. Molecular psychiatry. 2014;19(5):568-72.

6) Ambalavanan A, Girard SL, Ahn K, Zhou S, Dionne-Laporte A, Spiegelman D, et al. De novo variants in sporadic cases of childhood onset schizophrenia. Eur J Hum Genet. 2016;24(6):944-8.

7) Fernandez A, Drozd MM, Thümmler S, Dor E, Capovilla M, Askenazy F, et al. Childhood-Onset Schizophrenia: a systematic overview of its genetic heterogeneity from classical studies to the genomic era. Frontiers in Genetics. 2019;10:1137.

8) Berridge MJ. The inositol trisphosphate/calcium signaling pathway in health and disease. Physiological reviews. 2016;96(4):1261-96.

9) Timms AE, Dorschner MO, Wechsler J, Choi KY, Kirkwood R, Girirajan S, et al. Support for the N-methyl-D-aspartate receptor hypofunction hypothesis of 
schizophrenia from exome sequencing in multiplex families. JAMA psychiatry. 2013;70(6):582-90.

10) Fernandez A, Dor E, Maurin T, Laure G, Menard M-L, Drozd M, et al. Exploration and characterisation of the phenotypic and genetic profiles of patients with early onset schizophrenia associated with autism spectrum disorder and their first-degree relatives: a French multicentre case series study protocol (GenAuDiss). BMJ open. 2018;8(7):e023330.

11) Dor-Nedonsel E, Menard M-L, Fernandez A, Sakarovitch C, Fontas E, SalleCollemiche X, et al. Early-Onset Schizophrenia in a paediatric population of French psychiatric and medico-social care centres: A cross sectional study. Plos one. 2020;15(7):e0236241.

12) Kaufman J, Birmaher B, Brent D, Rao U, Flynn C, Moreci P, et al. Schedule for affective disorders and schizophrenia for school-age children-present and lifetime version (K-SADS-PL): initial reliability and validity data. Journal of the American Academy of Child \& Adolescent Psychiatry. 1997;36(7):980-8.

13) Lord C, Rutter M, Le Couteur A. Autism Diagnostic Interview-Revised: a revised version of a diagnostic interview for caregivers of individuals with possible pervasive developmental disorders. Journal of autism and developmental disorders. 1994;24(5):659-85.

14) Sheehan DV, Lecrubier Y, Sheehan KH, Amorim P, Janavs J, Weiller E, et al. The Mini-International Neuropsychiatric Interview (MINI): the development and validation of a structured diagnostic psychiatric interview for DSM-IV and ICD-10. The Journal of clinical psychiatry. 1998. 
15) Sheehan DV, Lecrubier Y, Sheehan KH, Amorim P, Janavs J, Weiller E, et al. The Mini-International Neuropsychiatric Interview (MINI): the development and validation of a structured diagnostic psychiatric interview for DSM-IV and ICD-10. The Journal of clinical psychiatry. 1998.

16) Wechsler D. WISC-V: Technical and interpretive manual: NCS Pearson, Incorporated; 2014.

17) Kay SR, Fiszbein A, Opler LA. The positive and negative syndrome scale (PANSS) for schizophrenia. Schizophrenia bulletin. 1987;13(2):261-76.

18) Andreasen NC. The Scale for the Assessment of Negative Symptoms (SANS): conceptual and theoretical foundations. The British journal of psychiatry. 1989;155(S7):49-52.

19) Tombaugh TN. Trail Making Test A and B: normative data stratified by age and education. Archives of clinical neuropsychology. 2004;19(2):203-14.

20) Cloninger CR, Przybeck TR, Svrakic DM, Wetzel RD. The Temperament and Character Inventory (TCI): A guide to its development and use. 1994.

21) Pélissolo A, Veysseyre O, Lépine J-P. Validation of a computerized version of the temperament and character inventory (TCI) in psychiatric inpatients. Psychiatry research. 1997;72(3):195-9.

22) Baron-Cohen S, Wheelwright S, Skinner R, Martin J, Clubley E. The autism-spectrum quotient (AQ): Evidence from asperger syndrome/high-functioning autism, malesand females, scientists and mathematicians. Journal of autism and developmental disorders. 2001;31(1):5-17.

23) Todorov T, Todorova A, Georgieva B, Mitev V. A unified rapid PCR method for detection of normal and expanded trinucleotide alleles of CAG repeats in huntington 
chorea and CGG repeats in fragile X syndrome. Mol Biotechnol. 2010 Jun;45(2):150-4. doi: 10.1007/s12033-010-9260-y. PMID: 20217280.

24) Tevy MF, Seyres D, Traina C, Perrin L, Capovilla M. Ndae1 expression and regulation in Drosophila embryos. PloS one. 2014;9(3):e92956.

25) Mitchell CA, Speed CJ, Nicholl J, Sutherland GR. Chromosomal mapping of the gene (INPP5A) encoding the 43-kDa membrane-associated inositol polyphosphate 5phosphatase to 10q26. 3 by fluorescence in situ hybridization. Genomics. 1996;31(1).

26) Laxminarayan KM, Chan BK, Tetaz T, Bird PI, Mitchell CA. Characterization of a cDNA encoding the 43-kDa membrane-associated inositol-polyphosphate 5phosphatase. Journal of Biological Chemistry. 1994;269(25):17305-10.

27) Ooms LM, Horan KA, Rahman P, Seaton G, Gurung R, Kethesparan DS, et al. The role of the inositol polyphosphate 5-phosphatases in cellular function and human disease. Biochemical Journal. 2009;419(1):29-49.

28) Yang AW, Sachs AJ, Nystuen AM. Deletion of Inpp5a causes ataxia and cerebellar degeneration in mice. Neurogenetics. 2015;16(4):277-85.

29) De Smedt F, Verjans B, Mailleux P, Erneux C. Cloning and expression of human brain type I inositol 1, 4, 5-trisphosphate 5-phosphatase High levels of mRNA in cerebellar Purkinje cells. FEBS letters. 1994;347(1):69-72.

30) Volpatti JR, Al-Maawali A, Smith L, Al-Hashim A, Brill JA, Dowling JJ. The expanding spectrum of neurological disorders of phosphoinositide metabolism. Disease models \& mechanisms. 2019;12(8):dmm038174.

31) Speed CJ, Neylon CB, Little PJ, Mitchell CA. Underexpression of the $43 \mathrm{kDa}$ inositol polyphosphate 5-phosphatase is associated with spontaneous calcium oscillations and 
enhanced calcium responses following endothelin-1 stimulation. Journal of cell science. 1999;112(5):669-79.

32) Windhorst S, Minge D, Bähring R, Hüser S, Schob C, Blechner C, et al. Inositol-1, 4, 5-trisphosphate 3-kinase A regulates dendritic morphology and shapes synaptic $\mathrm{Ca} 2+$ transients. Cellular Signalling. 2012;24(3):750-7.

33) Billcliff PG, Lowe M. Inositol lipid phosphatases in membrane trafficking and human disease. Biochemical Journal. 2014;461(2):159-75.

34) Glantz LA, Lewis DA. Decreased dendritic spine density on prefrontal cortical pyramidal neurons in schizophrenia. Archives of general psychiatry. 2000;57(1):65-73.

35) Hutsler JJ, Zhang H. Increased dendritic spine densities on cortical projection neurons in autism spectrum disorders. Brain research. 2010;1309:83-94.

36) Allison JH, Stewart MA. Reduced brain inositol in lithium-treated rats. Nat New Biol. 1971 Oct 27;233(43):267-8. doi: 10.1038/newbio233267a0. PMID: 5288124.

37) Berridge MJ, Downes CP, Hanley MR. Neural and developmental actions of lithium: a unifying hypothesis. Cel1.1989 Nov 3;59(3):411-9. doi: 10.1016/0092-8674(89)90026-3. PMID: 2553271.

38) Chiu CT, Chuang DM. Neuroprotective action of lithium in disorders of the central nervous system. Zhong Nan Da Xue Xue Bao Yi Xue Ban. 2011;36(6):461-476. doi:10.3969/j.issn.1672-7347.2011.06.001

39) Cumsky HJ, Costello CM, Zhang N, Butterfield R, Buras MR, Schmidt JE, et al. The prognostic value of inositol polyphosphate 5-phosphatase in cutaneous squamous cell carcinoma. Journal of the American Academy of Dermatology. 2019;80(3):626-32. e1. 
40) Sekulic A, Kim SY, Hostetter G, Savage S, Einspahr JG, Prasad A, et al. Loss of inositol polyphosphate 5-phosphatase is an early event in development of cutaneous squamous cell carcinoma. Cancer prevention research. 2010;3(10):1277-83.

41) Xu B, Roos JL, Dexheimer P, Boone B, Plummer B, Levy S, et al. Exome sequencing supports a de novo mutational paradigm for schizophrenia. Nature genetics. 2011;43(9):864-8.

42) Ganesh S, Ahmed P H, Nadella RK, More RP, Seshadri M, Viswanath B, et al. Exome sequencing in families with severe mental illness identifies novel and rare variants in genes implicated in Mendelian neuropsychiatric syndromes. Psychiatry and Clinical Neurosciences. 2019;73(1):11-9.

43) Marioni RE, McRae AF, Bressler J, Colicino E, Hannon E, Li S, et al. Meta-analysis of epigenome-wide association studies of cognitive abilities. Molecular psychiatry. 2018;23(11):2133-44.

44) Seeds AM, Sandquist JC, Spana EP, York JD. A molecular basis for inositol polyphosphate synthesis in Drosophila melanogaster. Journal of Biological Chemistry. 2004;279(45):47222-32.

45) Obregón FP, Papalardo C, Castro S, Guerberoff G, Cantera R. Putative synaptic genes defined from a Drosophila whole body developmental transcriptome by a machine learning approach. BMC genomics. 2015;16(1):694.

46) Voßfeldt H, Butzlaff M, Prüßing K, Charthaigh R-AN, Karsten P, Lankes A, et al. Large-scale screen for modifiers of ataxin-3-derived polyglutamine-induced toxicity in Drosophila. PLoS One. 2012;7(11):e47452. 
47) Szego MJ, Ma'n HZ. Whole genome sequencing as a genetic test for autism spectrum disorder: from bench to bedside and then back again. Journal of the Canadian Academy of Child and Adolescent Psychiatry. 2016;25(2):116.

48) Soden SE, Saunders CJ, Willig LK, Farrow EG, Smith LD, Petrikin JE, et al. Effectiveness of exome and genome sequencing guided by acuity of illness for diagnosis of neurodevelopmental disorders. Science translational medicine. 2014;6(265):265ra168-265ra168.

49) Richards S, Aziz N, Bale S, Bick D, Das S, Gastier-Foster J, et al. Standards and guidelines for the interpretation of sequence variants: a joint consensus recommendation of the American College of Medical Genetics and Genomics and the Association for Molecular Pathology. Genetics in medicine. 2015;17(5):405-23. 


\section{Figure legends}

\section{Figure. 1. Genealogical tree of the patient's family}

The propositus is a 17-year-old female with COS (black dot).

Schizophrenia spectrum disorder status: affected (red); unaffected (green); unknown (white)

CNV 10q26.3 status: DUP + = positive; DUP - = negative

\section{Figure. 2. Mapping of a chromosome 10 micro-microduplication «chr10:134,258,887-134,582,804»}

(A) Schematic representation of chromosome 10 according to NCBI build 37 (human genome 19).

(B) Known RefSew Genes in the duplicated chromosomal area.

(C) Summary of coding genes, encoded proteins, corresponding OMIM reference and brain expression (RPKM mean score) included in the microduplication.

OMIM : www.omim.org;

NCBI GENE : www.ncbi.nlm.nih.gov/gene

Appendix 1. 5PtaseI expression in Drosophila embryos. 5PtaseI expression patterns in embryos detected by in situ hybridization with TSA amplification followed by histochemistry. (A) Ventral view of a stage 15 embryo. Strong expression is observed in the vernal nerve cord. (B) Lateral view of a stage 15 embryo. Strong expression is observed in the brain and in the ventral nerve cord. (C) Lateral view of a stage 16 embryo. Strong expression is observed in the brain and in the ventral nerve cord. In all panels, the anterior end is to the left. 